\title{
Misdiagnosis of diabetic ketoacidosis as pelvic inflammatory disease in a Ghanaian teenager: A case report
}

Emmanuel Ameyaw ${ }^{1 *}$, Nana Ama Barnes ${ }^{2}$, Serwah Bonsu Asafo-Agyei ${ }^{1}$ and April Deborah Amakye Ansah ${ }^{1}$

${ }^{1}$ Department of Child Health, Komfo Anokye Teaching Hospital, P. O. Box 1934, Kumasi, Ghana

${ }^{2}$ Department of Internal Medicine Effia Nkwanta Regional Hospital, P O Box 229 Sekondi, Ghana

\begin{abstract}
Misdiagnosis of diabetic ketoacidosis (DKA) among children and adolescents with diabetes is often misdiagnosed in clinical practice in Ghana, and it occurs at primary, secondary and tertiary care settings and among all grades of health practitioners. This is because DKA mimics many other disorders such as infections, surgical and obstetric emergencies and these conditions are more frequently seen in hospitals in Ghana than diabetes. It is, therefore, paramount that every child or adolescent presenting to a health facility with any suspected acute disease to warrant admission is investigated, as well, for diabetes by checking blood glucose irrespective of the working diagnosis.
\end{abstract}

\section{Introduction}

Type 1 diabetes mellitus (T1D) is a heterogeneous disorder characterized by destruction of pancreatic beta cells, resulting in absolute insulin deficiency [1]. The majority of cases are attributable to an autoimmune-mediated destruction of beta cells (type 1a) while a small minority of cases results from idiopathic destruction or failure of beta cells (type 1b). T1D accounts for $5-10 \%$ of the total cases of diabetes [2]. However, more than $75 \%$ of DKA occurs in patients with T1D [3,4]. Given the huge numbers of patients with type 2 diabetes mellitus (T2D) in Ghana, over the years, less attention has been accorded to T1D [5]. The first Paediatric endocrine Clinic (PEC) in Ghana, giving supervised care exclusively to children and adolescents with diabetes and other endocrine disorders, was established at Komfo Anokye Teaching Hospital (KATH), Kumasi, Ghana in January, 2012 after a successful training of a paediatric endocrinologist at the Paediatric Endocrinology Training Center for Africa (PETCA) in Nairobi, Kenya. Since inception of the clinic misdiagnosis of diabetes and other endocrine disorders among children and adolescents have frequently been encountered, the consequences of some of which have been fatal. DKA can easily be misdiagnosed because clinical features usually mimic infectious diseases [2-4], surgical emergencies such as acute abdomen, bowel perforation, appendicitis and ruptured ectopic pregnancy [6-8]. DKA is, potentially, a life threatening acute complication of T1D which is an important cause of mortality and morbidity in Africa [3,4]. The true incidence of T1D and DKA in Ghana and many other African countries is not known [4,9]. Misdiagnosis of DKA will lead to mismanagement which may result in complications including mortality. We present a 19 year old Ghanaian with DKA who presented to a hospitals in Ghana but was misdiagnosed and as a result ended in a mortality. No such previous report was found in the literature.

\section{Case report}

A 19 year old female presented to a Regional Hospital with severe abdominal pain of acute onset associated with vomiting. History was difficult to elicit as she was in severe pain. Accompanied relative claimed the patient had lost weight within the past two months.

On examination she was extremely restless with no remarkable abdominal findings. A rapid urine pregnancy test was negative.

An initial diagnosis of pelvic inflammatory disease was made and she was started on IV metronidazole and ciprofloxacin.

On review she was unconscious, pulse was 110 /minute, BP was $70 / 33 \mathrm{mmHg}$. Diagnosis was changed to septic shock secondary to pelvic inflammatory disease and the conclusion was to continue IV antibiotics and fluid resuscitation. A gyanaecologist reviewed after one hour and found her conscious but restless, pulse was104/minute, regular but weak volume, BP was 70/33 mmHg. Blood glucose could not be done because of lack of glucose strips. Vaginal examination was normal except for small brownish discharge. IV gentamycin, ceftriaxone, clindamycin and $4 \mathrm{~L}$ normal saline, $2 \mathrm{~L}$ of $5 \%$ Dextrose Saline were ordered.

The next morning her blood glucose level was $30.7 \mathrm{mmol} / \mathrm{l}$ and urine ketones was large. The diagnosis was then changed to DKA. She was then managed with normal saline and hourly intramuscular regular insulin. There was no urine production so potassium was withheld until she could pass urine.

Laboratory tests such as electrolytes, urea and creatinine were requested but they could not be done as the laboratory did not have the required reagents.

Patient was reviewed the same day in the evening by physicians, random blood glucose was $24.0 \mathrm{mmol} / \mathrm{l}$, GCS was $13 / 15$ blood pressure

Correspondence to: Emmanuel Ameyaw, Department of Child Health, Komfo Anokye Teaching Hospital, P. O. Box 1934, Kumasi, Ghana, Tel: +233208403784 E-mail: ekameyaw@yahoo.com

Key words: Type 1 diabetes, diabetic ketoacidosis, misdiagnosis, children and adolescents

Received: April 02, 2017; Accepted: April 26, 2017; Published: April 29, 2017 
was $67 / 40 \mathrm{mmHg}$ with no recorded urine output. Instruction was given to monitor urine output and increase the amount of intravenous fluid as well as fluids via nasogastric tube.

Patient died after spending about 38 hours in the hospital.

\section{Discussion}

DKA is a medical emergency that requires treatment and monitoring for multiple metabolic abnormalities and vigilance for complications. It is the leading cause of mortality and permanent disability in children and adolescents with T1D [3,4]. In Africa the mortality of DKA is unacceptably high with a reported death rate of 26 to $29 \%$ according to studies done in Kenya, Tanzania and Ghana $[2,5,10]$. Misdiagnosis of DKA can be lethal as the patient so misdiagnosed would be mismanaged and hence would develop further complications and may even end up in mortality $[4,7,8,10]$.

Clinical features of DKA mimic many conditions and can, therefore, result in misdiagnosis. The patient was seen and reviewed by a number of specialists but they all failed to diagnose DKA but rather made various diagnoses such as acute PID, sepsis and septic shock which all mimic DKA in terms of clinical features. Although shock was appropriately diagnosed, it was not due to septicaemia or PID but rather severe dehydration from polyuria due to undiagnosed diabetes. She developed acute kidney injury which was not recognized and, therefore, was not managed. The patient died as a result of inappropriate management due to misdiagnosis of DKA. Part of management of severe infections such as severe PID, septicaemia, cerebral malaria, pneumonia includes dextrose administration. A DKA patient erroneously given dextrose would develop cerebral edema and if not recognized and appropriately managed could die.

Diabetes mellitus should be suspected in any child or adolescent with acute medical and/or surgical condition on admission in a hospital $[4,6,8,10]$.

\section{Conclusion}

DKA among children in Ghana can easily be misdiagnosed because clinical features can mimic other acute diseases. Laboratory support is nonexistent or poorly available in many hospitals in Ghana. Many hospitals do not readily have glucose meters and strips and so checking blood glucose is usually omitted. It is likely that children and adolescents with diabetes are commonly being misdiagnosed as infections. It is, therefore, important that all doctors, irrespective of discipline and level of practice, whether in primary, secondary or tertiary care institutions, should be able to diagnose DKA and initiate appropriate management to improve upon chances of survival.

\section{Recommendation}

Any child or adolescent with acute disease should have blood glucose tested to rule out diabetes. Every hospital or clinic should have glucose meters and strips for checking blood glucose of patients.

\section{Funding statement}

This research did not receive any grant.

\section{Conflict of interest}

No conflict of interest.

\section{Acknowledgement}

We are grateful to Dr. Jean-Pierre Chanoine, Head of Endocrinology and Diabetes Unit, BC Children's Hospital, Vancouver.

\section{References}

1. Maahs DM, West NA, Lawrence JM, Mayer-Davis EJ (2010) Epidemiology of type 1 diabetes. Endocrinol Metab Clin North Am 39: 481-497. [Crossref]

2. Hall V, Thomsen RW, Henriksen O, Lohse N (2011) Diabetes in Sub Saharan Africa 1999-2011: epidemiology and public health implications. A systematic review. BMC Public Health 11: 564. [Crossref]

3. Van Zyl DG (2008) Diagnosis and treatment of diabetic ketoacidosis. S Afr Fam Pract 50: 35-39.

4. Murunga AN, Owira PM (2013) Diabetic ketoacidosis: an overlooked child killer in sub-Saharan Africa? Trop Med Int Health 18: 1357-1364. [Crossref]

5. J Kratzer (2012) Structural barriers to coping with type 1 diabetes mellitus in Ghana Experiences of Diabetic Youth and their families. Gh Med J 46: 39-45.

6. Durai R, Hoque H, Ng P (2010) The Acute Abdomen-Commonly Missed and Misdiagnosed Conditions: Review. Webmed Central Surgery 1: 1-14.

7. Glynn M, Elliot D (1984) Diabetic ketoacidosis and ruptured ectopic pregnancy: a fatal combination. Br Med J 288: 1287-1288.

8. Huang FY, Huang SH, Hsu CH (1990) [Abdominal pain in diabetic ketoacidosis: report of four cases]. Zhonghua Min Guo Xiao Er Ke Yi Xue Hui Za Zhi 31: 191-195. [Crossref]

9. Onyiriuka AN, Ifebi E (2013) Ketoacidosis at diagnosis of type 1 diabetes in children and adolescents: frequency and clinical characteristics. J Diabetes Metab Disord 12: 47-52.

10. Rwiza HT, Swai ABM, McLarty DG (1986) Failure to diagnose diabetic ketoacidosis in Tanzania. Diabet Sci 3: 181-183.

Copyright: (C2017 Ameyaw E. This is an open-access article distributed under the terms of the Creative Commons Attribution License, which permits unrestricted use, distribution, and reproduction in any medium, provided the original author and source are credited. 